\title{
Transient-gain photoionization x-ray laser
}

\author{
Clemens Weninger and Nina Rohringer \\ Max Planck Institute for the Physics of Complex Systems, 01187 Dresden, Germany \\ and Center for Free Electron Laser Science, 22607 Hamburg, Germany
}

(Received 14 October 2014; published 22 December 2014)

\begin{abstract}
We present a generalized theory based on one-dimensional Maxwell-Bloch equations to study the amplification process of an inner-shell photoionization-pumped atomic x-ray laser. Focusing an x-ray free-electron laser beam in an elongated neon-gas target results in a strong exponential amplification of $K \alpha$ fluorescence, as recently demonstrated [N. Rohringer et al., Nature (London) 481, 488 (2012); C. Weninger et al., Phys. Rev. Lett. 111, 233902 (2013)]. Here, we present an in-depth theoretical study of the amplification process that goes beyond the previous theory based on a rate-equation approach. We study the evolution of the pulse characteristics during the amplification process for transform-limited Gaussian and broadband self-amplified spontaneous-emission pump pulses. We discuss the impact of the gain-dependent group velocity on the emitted x-ray radiation and the resulting gain-guiding effects. A thorough analysis of the spectral and temporal properties of the emitted radiation is presented, including higher-order field-correlation functions, to characterize the ensemble of emitted $\mathrm{X}$-ray pulses.
\end{abstract}

DOI: 10.1103/PhysRevA.90.063828

PACS number(s): 42.55.Vc, 32.30.Rj, 42.65.Dr, 78.70.Ck

\section{INTRODUCTION}

Irradiating atoms with $\mathrm{x}$ rays that are tuned above the ionization edge of inner shells leads to short-lived core-excited states by photoionization. These core-excited ions decay predominantly via fast Auger decay for light atoms, with typically small fluorescence yields. When the X-ray intensities are large enough, so that ionization rates become comparable to the Auger-decay rates, a sizable population inversion between inner-shell holes and occupied valence shells can be achieved. This population inversion can, in turn, lead to the buildup of a macroscopic polarization of the target and stimulated emission. As recently demonstrated in two experimental campaigns [1,2] at the Linac coherent light source (LCLS) $\mathrm{X}$-ray free-electron laser (XFEL), this can result in saturated amplification on an inner-shell transition (a single-pass $\mathrm{x}$-ray amplifier), with exponential gain factors on the order of 20 , corresponding to an increase of irradiated $\mathrm{x}$-ray energy of 8 orders of magnitude. Here, we present an in-depth theory study of this process that goes beyond the previous approaches that were based on rate equations.

X-ray lasers (XRLs) were first proposed in the 1960s [3], and since then, different pumping schemes have been studied in theory and experiment [4-6], which led to the first demonstrations of laboratory x-ray lasers in 1984 [7,8]. The photoionization inner-shell $\mathrm{x}$-ray laser scheme was originally proposed by Duguay and Rentzepis [3], and since then, several possible targets and pumping schemes were studied in theory [9-16]. Although experimental attempts have been made and an inner-shell laser was demonstrated in the blue spectral domain [17] in 1983 and in the EUV region by Auger-decay pumping [18], the development of powerful XFELs [19-23] was needed to demonstrate this scheme in the soft X-ray spectral region at $1.45-\mathrm{nm}$ wavelength by a laboratory-based experiment [1].

In the following we will perform an in-depth theoretical study of our recently demonstrated amplified spontaneous $\mathrm{X}$-ray emission [1,2] following pumping of neon gas at 500 Torr pressure with an XFEL pulse tuned above the $K$ edge.
In contrast to previously published studies, which considered pumping the Rydberg resonances in the pre- $K$-edge region of neon [2,24], here, we focus on pure photoionization pumping with photon energies well above the $K$ edge of neon $(870.2 \mathrm{eV})$. We will demonstrate that both the spectral and temporal characteristics of the irradiated $\mathrm{x}$-ray radiation differ considerably in the case of resonantly versus photoionizationpumped X-ray lasers. The majority of previous theoretical studies were based on small-signal gain estimates based on a rate-equation approach, and only a few considered possible propagation effects, which were discussed under the terminology of laser lethargy and coherence brightening $[25,26]$. In these approaches, a fully quantum-mechanical description for both matter and the electromagnetic field was employed in order to correctly treat spontaneous emission. The resulting operator equations were solved numerically for a swept-gain amplifier restricted to the linear regime, assuming instantaneous pumping. Here, we show the results of a semiclassical generalized Maxwell-Bloch approach, in which the field is treated classically and the gain medium is treated by a quantum-mechanical approach limited to the involved atomic and ionic bound states.

\section{THEORETICAL APPROACH}

To model the amplified x-ray emission a one-dimensional semiclassical Maxwell-Bloch model [27,28] is used. In contrast to the rate-equation approach [15] the ionic system is treated quantum mechanically by the density-matrix formalism. This allows us to calculate the spectral and coherence properties of the emitted $\mathrm{x}$-ray radiation, which is not possible in the rate-equation approach without additional assumptions.

The initially neutral neon atoms are photoionized by the focused XFEL beam, which is tuned well above the neon $K$ edge $(870.2 \mathrm{eV})$. Since the photoionization of the valence shell for these high-photon energies is an order of magnitude smaller than ionization of the inner shell, this results mainly in the population of the core-excited state $|1 s\rangle=\left|1 s^{1} 2 s^{2} 2 p^{6}\right\rangle$ 


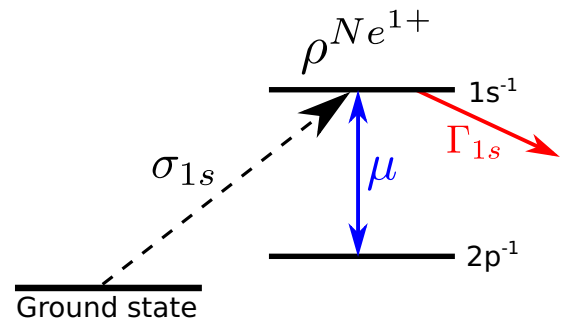

FIG. 1. (Color online) Level scheme for neon. The initially neutral neon ground state is depleted by photoionization, and predominantly, the $1 s^{-1}$ state (upper lasing state) is populated (dotted arrow). This state is dipole coupled to the lower lasing state $2 p^{-1}$ (blue arrow). The red arrow indicates the loss term due to the Auger decay.

(upper level of the lasing transition). This state predominantly decays via Auger decay according to the Auger rate $\Gamma_{1 s}=$ $1 / 2.4 \mathrm{fs}^{-1}$, thereby populating states of the doubly charged neon ion. Alternatively, the state can decay radiatively to the ionic ground state $|2 p\rangle=\left|1 s^{2} 2 s^{2} 2 p^{5}\right\rangle$ (lower level of the lasing transition), as shown in Fig. 1. The fluorescence yield in neon is only $\approx 2 \%$. Considering the level fine structure, the lower states of the lasing transition correspond to the doublet $2 p^{-12} P_{1 / 2}, 2 p^{-12} P_{3 / 2}$. The fine-structure splitting of these states amounts to $0.11 \mathrm{eV}$, which is smaller than the Auger width of $\Gamma_{1 s}=0.27 \mathrm{eV}$, which determines the natural linewidth of the transition. For simplicity we only treat a single final state in the following discussion.

The radiation is treated classically and split into the pumping radiation (XFEL) and the emitted x-ray radiation. The pumping radiation is treated as a photon flux; that is, no wave equation is solved for the XFEL pulse. This is a good approximation since we assume that the pulse is tuned far above any resonances and the ionization cross section can be assumed to be constant over the XFEL bandwidth. The XFEL pulse is strongly attenuated in the medium, so that the pump flux is determined by the following equation:

$$
\frac{\partial J_{p}(\tau, z)}{\partial z}=-\rho_{0}(\tau, z) n\left(\sigma_{1 s}+\sigma_{v}\right) J_{p}(\tau, z) .
$$

Here, $n$ is the density of neon atoms, $\rho_{0}$ is the groundstate occupation probability, and $\sigma_{1 s}$ and $\sigma_{v}$ denote the $1 s$ and valence photoionization cross sections, respectively. The ground-state occupation $\rho_{0}$ therefore evolves as

$$
\frac{\partial \rho_{0}(\tau, z)}{\partial \tau}=-\left(\sigma_{1 s}+\sigma_{v}\right) J_{p}(\tau, z) \rho_{0}(\tau, z) .
$$

The emitted x-ray radiation, on the other hand, is treated as an electric field. This is necessary, as will be demonstrated, since propagation in the high-gain medium will have a strong impact on the group velocity of the propagating $x$-ray field. The electric field is decomposed into a complex slowly varying envelope $\mathcal{E}$ and positive and negative frequency components,

$$
E(t)=\frac{1}{2}\left[\mathcal{E}(t, z) e^{-i \omega_{L} t}+\mathcal{E}^{\star}(t, z) e^{i \omega_{L} t}\right],
$$

where $\omega_{L}$ denotes the transition frequency. Inserting this decomposition into the wave equation for the electric field and making use of the slowly varying envelope approximation results in a first-order equation for the envelope $\mathcal{E}$. This equation can be further simplified by transforming it in a frame moving at the speed of light using the retarded time $\tau=t-\frac{z}{c}$. This transformation makes the radiation almost stationary in this frame and avoids the numerical problem of dispersion when propagating over long distances [29]. The final equation for the field envelop reads

$$
\frac{\partial \mathcal{E}(\tau, z)}{\partial z}=i \frac{2 \pi \omega_{L}}{c} \mathcal{P},
$$

where $c$ is the vacuum speed of light and $\mathcal{P}$ is the slowly varying envelope of the macroscopic polarization of the medium.

The reduced ionic density matrix $\rho$ is coupled to the Maxwell equation via the slowly varying envelope of the polarization

$$
\mathcal{P}=2 n \mu \rho_{1 s 2 p},
$$

where $\mu=\langle 1 s|z| 2 p\rangle=6.8 \times 10^{-2}$ is the electric dipole moment between the $1 s$ and $2 p$ orbitals. The reduced ionic density matrix describes the occupations and coherence of the core-ionized intermediate state $|1 s\rangle$ and the ionic ground state $|2 p\rangle$. The equation of motion for the reduced ionic density matrix can be derived starting with the Schrödinger equation of the full $N$-electron system. The ejected photoelectron is not observed. This results in the definition and then equation of motion of the reduced ionic density matrix $[24,30]$ :

$$
\begin{aligned}
& \frac{\partial \rho_{1 s 1 s}(\tau, z)}{\partial \tau}=-\Gamma_{1 s} \rho_{1 s 1 s}+\sigma_{1 s} J_{p} \rho_{0}-\mu \operatorname{Im}\left(\mathcal{E} \rho_{2 p 1 s}\right), \\
& \frac{\partial \rho_{2 p 2 p}(\tau, z)}{\partial \tau}=\sigma_{2 p} J_{p} \rho_{0}+\mu \operatorname{Im}\left(\mathcal{E} \rho_{2 p 1 s}\right), \\
& \frac{\partial \rho_{2 p 1 s}(\tau, z)}{\partial \tau}=-\frac{\Gamma_{1 s}}{2} \rho_{2 p 1 s}+i\left(\rho_{1 s 1 s}-\rho_{2 p 2 p}\right) \frac{\mu \mathcal{E}^{\star}}{2}+S .
\end{aligned}
$$

Here, we added a phenomenological random source term $S$ to model spontaneous emission that acts as a seed for the amplification process. The source term is modeled as Gaussian white noise with the following correlation function:

$$
\begin{aligned}
\left\langle S\left(t_{1}\right) S^{\star}\left(t_{2}\right)\right\rangle & =F \delta\left(t_{1}-t_{2}\right), \\
F & =\frac{c^{2} \rho_{1 s 1 s} \gamma_{r} \hbar \Omega \Gamma_{1 s}^{2}}{4 \pi^{2} \omega_{L} \mu},
\end{aligned}
$$

where $\gamma_{r}$ is Einstein's $A$ coefficient for spontaneous emission and $\Omega$ is the solid angle in the forward direction over which an inverted medium can be achieved. In our case, $\arctan (\Omega) \sim d / L$, where $d$ is the focus size and $L$ is the length of the target, assuming a pencil-shaped inverted target. The correlation function of Eq. (7) produces the correct Lorentzian line shape for spontaneous emission [27]. The source term is properly normalized to inject energy in the system with the spontaneous fluorescence rate. Equations (1)-(6) are solved self-consistently and propagated through space to describe the amplification of spontaneous emission. Equations (1) and (4) are solved by a second-order Adams-Bashforth method. The atomic system is solved by a split-step method [24,31] with integration steps $\Delta t=0.01 \mathrm{fs}$ and $\Delta z=c \Delta t$.

\section{GAUSSIAN PUMP PULSE}

In the following we study the amplification process for a temporally Gaussian pump pulse. With the advance of 


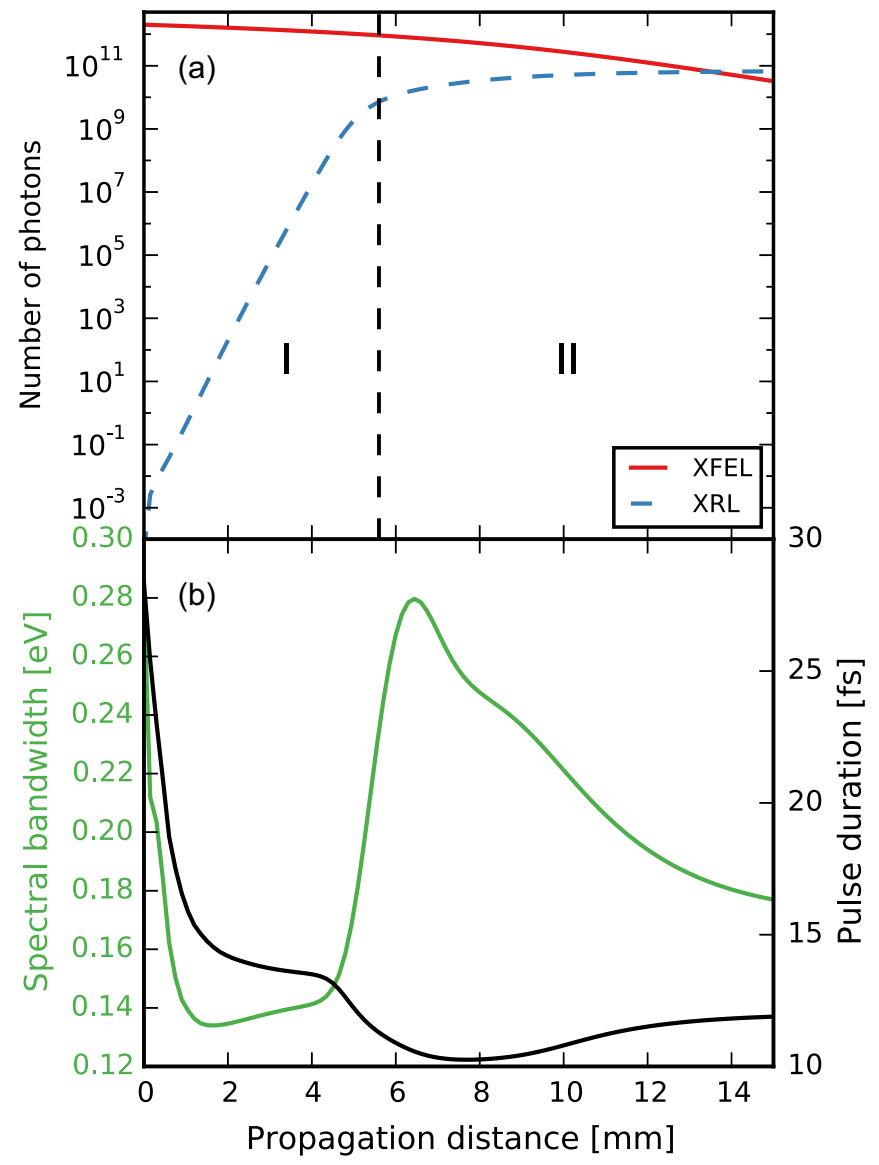

FIG. 2. (Color online) Gain curve and evolution of the spectral width and pulse duration. (a) The number of photons in the XFEL (red solid line) and the XRL (blue dashed line) as a function of propagation distance. The black dashed vertical line separates the linear gain region I from the saturated gain region II. (b) The spectral width [FWHM, green (gray) line] and the pulse duration (FWHM, black line) as a function of propagation distance. The XFEL pulse parameters are 40 -fs pulse duration, $2 \times 10^{12}$ photons per pulse, and $2-\mu \mathrm{m}$ focal radius, and we assumed a gas density of $1.6 \times 10^{19} \mathrm{~cm}^{-3}$ according to the parameter range of previous experiments.

seeded XFEL sources [22] and so-called self-seeded FELs [32] spectrally stable and longitudinally coherent $\mathrm{x}$-ray radiation becomes accessible. Figure 2(a) shows the expected number of photons of the XFEL pump and the XRL emission as a function of the propagation distance. In accordance with our recent experiment [1] we assume $2 \times 10^{12}$ photons per pulse, a pulse duration of $40 \mathrm{fs}$ (FWHM of the intensity), and a focus radius of $2 \mu \mathrm{m}$. The gas density of the medium is set to $10^{19} \mathrm{~cm}^{-3}$, corresponding to 485 Torr pressure, and we assume a total length of $15 \mathrm{~mm}$ for the gas medium. The number of photons in the XRL initially increases exponentially. At $6 \mathrm{~mm}$ the slope of the XRL gain curve decreases, and the amplification is no longer exponential, a sign of saturation. At the end of the medium the XRL reaches $7 \times 10^{10}$ photons, corresponding to an integrated gain-length product of 21 , assuming exponential amplification from the initial spontaneous emission. The XFEL pump pulse is strongly absorbed in the medium. Initially, the medium is bleached, and saturated absorption of the pump pulse leads to a smaller absorption length.
Propagating through the medium, the pump intensity gradually decreases, resulting in longer rise times of the population inversion, and the ionization degree of the target decreases. The total transmission of the XFEL pulse after passing through the medium amounts to $2 \%$. Figure 2(b) shows the pulse duration and spectral width of the amplified spontaneous X-ray emission as a function of the propagation distance. The XRL pulse starts out with a bandwidth of $0.27 \mathrm{eV}$, corresponding to the natural lifetime of the upper state. Then a quick drop in the spectral bandwidth is observable as the exponential amplification sets in. In most of the exponential gain region (linear gain regime) the bandwidth stays almost constant. The pulse duration shows a similar behavior in the linear gain regime. The initial pulse duration is approximately $30 \mathrm{fs}$, which corresponds to the duration of the population inversion of the medium at short propagation distances. Evidently, the emitted x-ray pulse is initially not transform limited. With propagation the temporal duration of the $\mathrm{x}$-ray pulse drops down and then stays almost constant and reaches the transform-limited pulse duration, up to the point when saturation sets in. The amplification behavior of this transient $\mathrm{x}$-ray laser is quite different from the usual spectral gain narrowing in amplified spontaneous-emission lasers [33,34]. Initially, gain narrowing is observed, but then it is followed by a long amplification period of nearly constant spectral and temporal width and the buildup of soliton-like pulses. At saturation the emission line features the typical saturation broadening, and the pulse duration drops.

The evolution of the XRL spectrum with propagation and three spectra for different positions in the medium are shown in Fig. 3. At the beginning of the medium the spectrum has a Lorentzian shape, determined by the spectral properties of spontaneous emission. The exponential decay of the upper state leads to the Lorentzian shape and shows that the mechanism for modeling the spontaneous emission works well and produces the correct spectral characteristics. With propagation the spectrum first becomes narrower and loses the Lorentzian tails. The spectrum quickly broadens as saturation

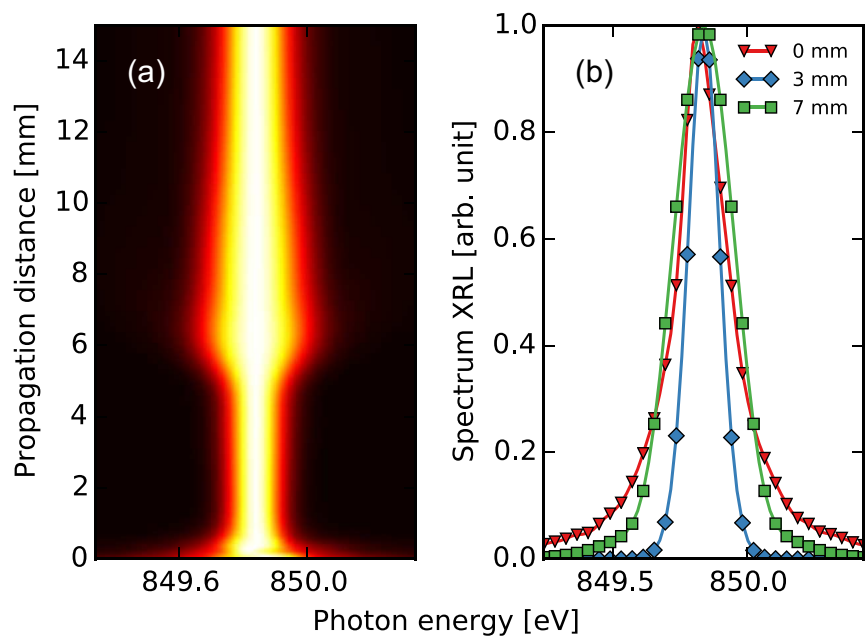

FIG. 3. (Color online) (a) Evolution of the XRL spectra as a function of propagation distance. (b) Normalized XRL spectra at three different points in the medium with FWHM bandwidths of $0.27,0.14$, and $0.28 \mathrm{eV}$, respectively. 

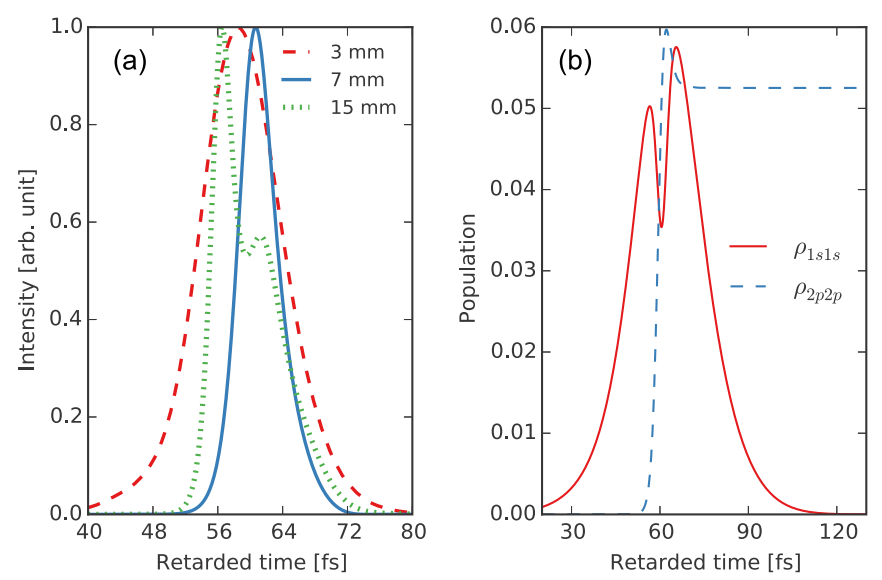

FIG. 4. (Color online) (a) Normalized XRL temporal pulse shape at three different points in the medium. (b) Population of state $\rho_{1 s 1 s}$ and the final state $\rho_{2 p 2 p}$ after $7 \mathrm{~mm}$ of propagation.

sets in and reaches its maximal bandwidth. The temporal pulse shape in Fig. 4(a) initially shows a single intensity spike. The pulse duration of the single spike has its minimum at $7 \mathrm{~mm}$ of propagation. At this distance the peak intensity corresponds to the saturation intensity of the laser, i.e., the intensity for which the stimulated emission rate equals the Auger-decay rate. The trailing edge of the pulse, below the saturation intensity, still gets amplified in the medium, leading to the appearance of a new peak. The evolution of the XRL temporal shape is depicted in Fig. 5(a). Figure 4(b) shows the population of the core-excited state and the final state at the point of saturation (roughly at a propagation distance of $7 \mathrm{~mm}$ ). The populations show one damped Rabi oscillation between the core-excited state $\rho_{1 s 1 s}$ and the final state $\rho_{2 p 2 p}$.

Considering the final-state doublet $2 p^{-12} P_{1 / 2}, 2 p^{-12} P_{3 / 2}$ instead of a single final state does not have a big impact on the amplification process. When the two final states are included, the integrated gain does not change considerably since spacing of the levels is smaller than the lifetime width of the upper lasing state. We find, however, a different evolution of the spectral line shape, shown in Fig. 6(b). Figure 6(a) shows that there is significantly less spectral broadening in saturation for the final-state doublet than for the case with a single final state. For the final-state doublet the photons are split between the two transitions, leading to a lower intensity on each transition and therefore less spectral broadening. In the following discussions we will stick to the case of a single emission level to highlight the details of the amplification process. Adding the fine structure of the final states does not alter any of the conclusions and gives only slightly different results.

In the following the amplification process is discussed in more detail (see Fig. 5, where the normalized temporal shape of the XFEL pump pulse, the population inversion, and the temporal irradiated XRL intensity profile are shown as a function of the propagation distance. The propagation of the pump pulse through the optically dense medium shown in Fig. 5(c) results in two effects that are important for the transient gain: Due to bleaching of the medium (saturated absorption), the absorption of the pump pulse results in a reshaping of the temporal pulse profile. That is, mainly, the leading edge of the pulse is absorbed in the medium. This
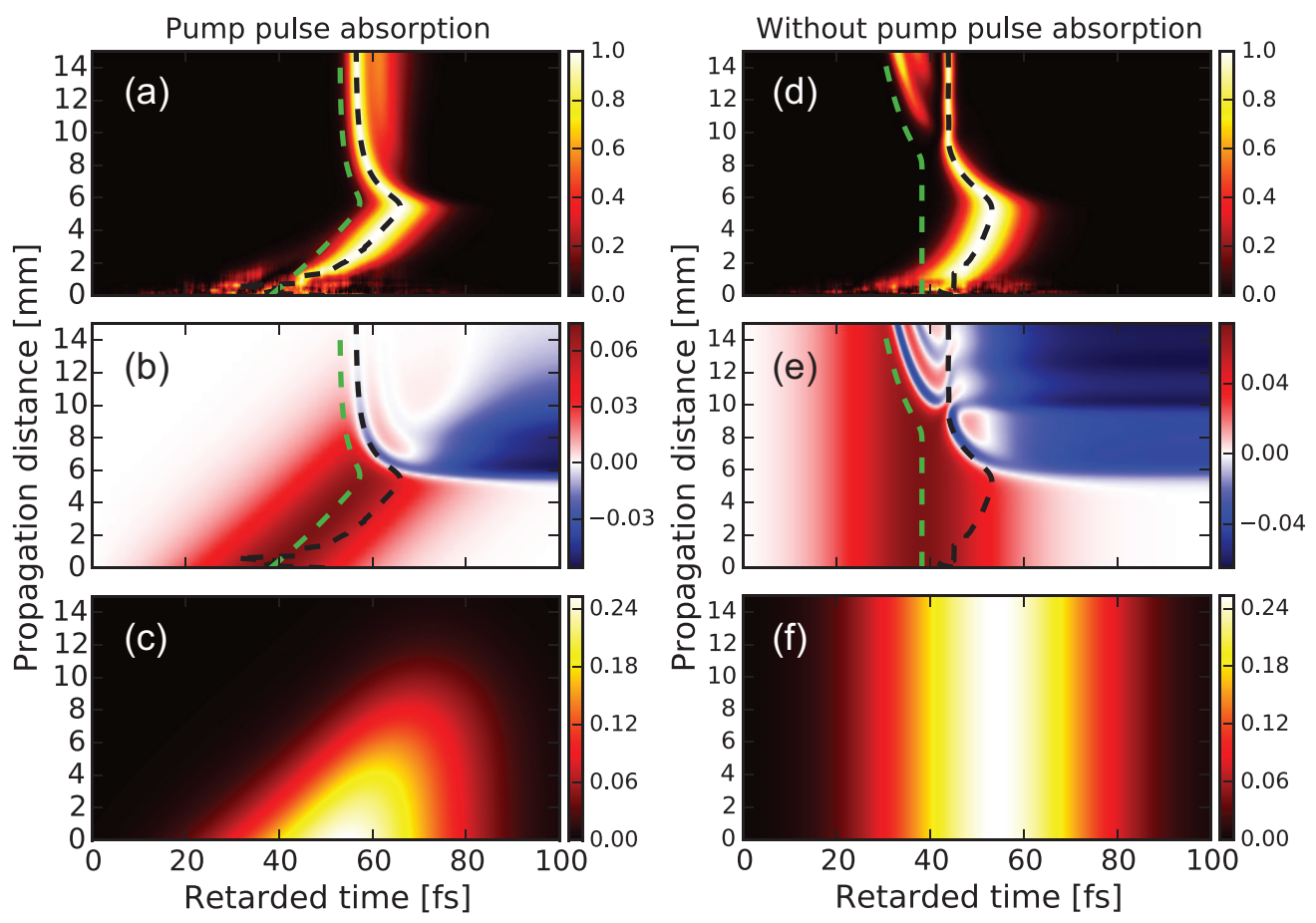

FIG. 5. (Color online) Evolution of (a) and (d) the normalized temporal XRL profile, (b) and (e) the population inversion, and (c) and (f) the XFEL pump pulse as a function of propagation depth. The figures on the left include pump pulse absorption, while the ones on the right are without pump pulse absorption. The black dashed line follows the maximum of the XRL intensity, while the green (gray) dashed line follows the maximum of the population inversion. In the retarded frame the maximum XFEL pump intensity is initially at $40 \mathrm{fs}$. 

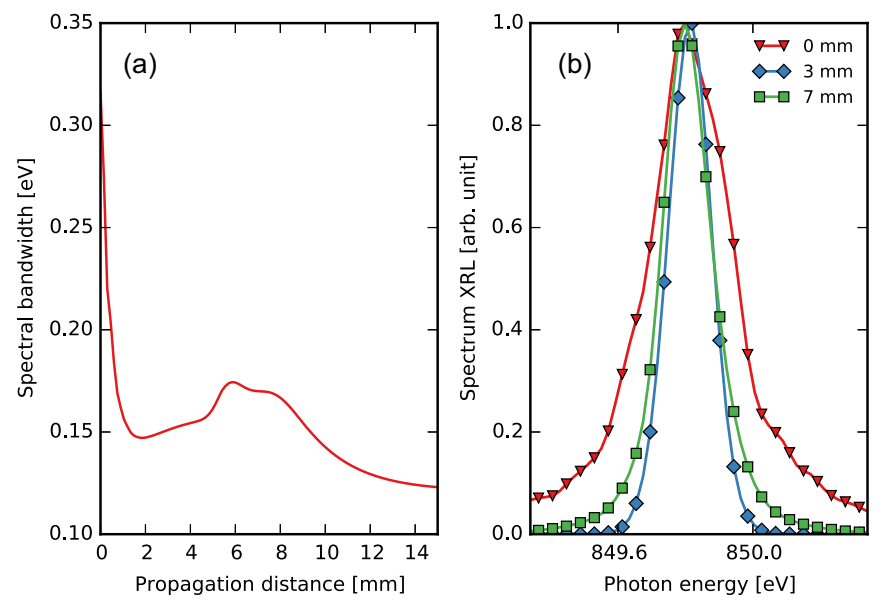

FIG. 6. (Color online) (a) Evolution of the spectral bandwidth, (b) XRL spectra at different positions in the medium with bandwidths of $0.27,0.15$, and $0.17 \mathrm{eV}$, respectively, for the final-state doublet, supposing pump-parameters in Fig. 2.

leads to a steepening of the leading edge of the pump pulse. Moreover, the pulse maximum is shifted backwards in the frame of the propagating pulse. The change in the temporal shape of the pump pulse is also imprinted on the temporal profile of the population inversion in the medium. With increasing propagation distance, the peak of the population inversion is pushed backwards in time. In other words, the peak of the pump pulse and the population inversion travel with a velocity smaller than the speed of light, as can be seen in Figs. 5(b) and 5(c).

Another critical aspect of the evolution of gain concerns the group velocity of the emitted XRL radiation. Typically, for a pulse propagating on a high-gain resonant transition the group velocity is slower than the speed of light [35]. This can often lead to a velocity mismatch (slippage) between the population inversion and the propagating XRL radiation, leading to a drastic reduction in the gain of transient $\mathrm{X}$-ray laser schemes [36,37]. We will, however, see that the absorption of the pump pulse along with the reduction of the group velocity of the emitted radiation will lead to a gain-guiding effect that guarantees that population inversion and the amplified x-ray pulses overlap in time throughout the exponential gain region. In the retarded time frame vertical lines move at the speed of light, while lines moving to the right move at a lower velocity. As can be seen in Figs. 5(a)-5(c) the emitted XRL radiation, the population inversion, and the pump pulse propagate with a group velocity smaller than the speed of light $\left(0.998^{*} c\right)$ and show a clear temporal overlap in the exponential gain regime (for distances $<7 \mathrm{~mm}$ ). The maxima of the pump pulse, the population inversion (green dashed line), and the maximum of the amplified XRL pulse (black dashed line), however, do not perfectly overlap. This reflects the necessary time for building up a macroscopic polarization of the medium starting from the population inversion. Analyzing the buildup of the polarization by solving Eq. (6) assuming a steplike increase of the population inversion followed by an exponential decay with the Auger rate, a delay time of the polarization maximum of $1.38 / \Gamma$ with respect to the maximum of the population inversion is found. The population inversion follows the XFEL pump intensity until saturation. At a propagation distance of approximately $7 \mathrm{~mm}$ the XRL is saturated and propagates with the speed of light through the medium.

Absorption of the pump pulse is essential for maintaining a high gain. For comparison and to highlight the gain-guiding principle, we also present results neglecting the pump-pulse absorption in the medium [Figs. 5(d)-5(f)]. In the absence of pump-pulse absorption a large temporal delay of the population inversion and the XRL pulse can be seen, resulting in a considerable reduction of the gain. Due to the lack of absorption of the pump pulse the amplification of the line emission eventually saturates, and the typical temporal and spatial ringing phenomenon of a saturated pulse propagating through the medium is observed in our simulations. Neglecting the pump absorption basically yields a swept-gain amplifier (the population inversion is created quasi-instantaneously and travels at the speed of light). This swept-gain amplifier has been studied previously [38,39]. The absence of a gain-guiding mechanism in this scheme, the fast decay of the core-excited state, and the finite amplifier bandwidth reduces the gain compared to small signal gain calculations based on rate equations.

\section{THE RATE EQUATION APPROACH}

Since all of the previous theoretical studies of transient X-ray laser schemes, with the exception of Refs. [38,39], were based on the rate-equation approach, which typically does not include effects of gain-dependent group velocity, it is useful to compare the rate-equation approach $[1,15]$ to the Maxwell-Bloch approach. In the rate-equation approach only the occupations of the involved lasing levels are treated, and the emitted radiation is treated as a time-dependent intensity. Amplification of the time-dependent intensity profile is directly determined by the population inversion and

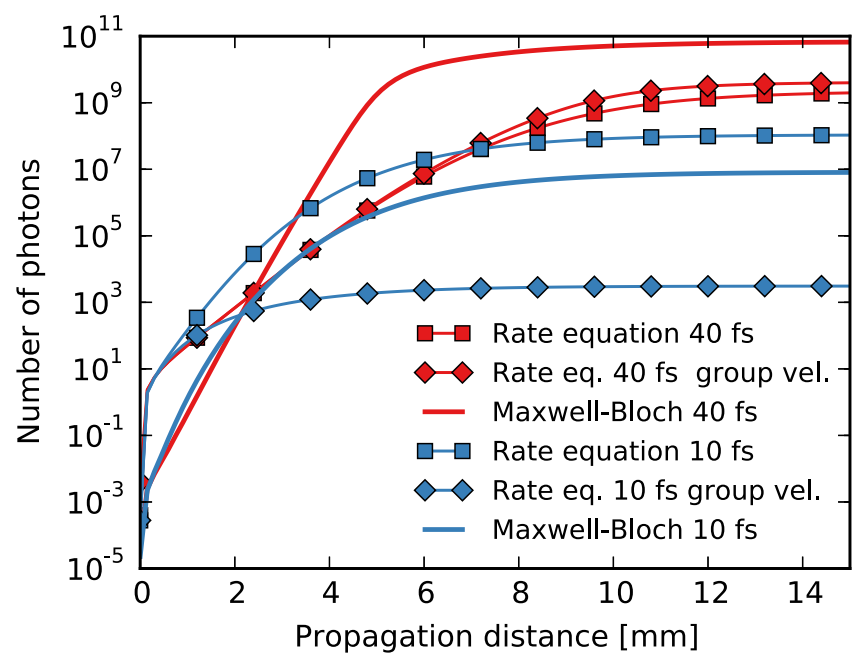

FIG. 7. (Color online) Comparison between the rate equation and the Maxwell-Bloch approach for a Gaussian pump pulse with 10and 40 -fs duration with $5 \times 10^{11}$ and $2 \times 10^{12}$ photons, respectively, resulting in the same peak intensity. Shown are the gain curves, i.e., the number of emitted photons as a function of propagation distance through the gain medium. 

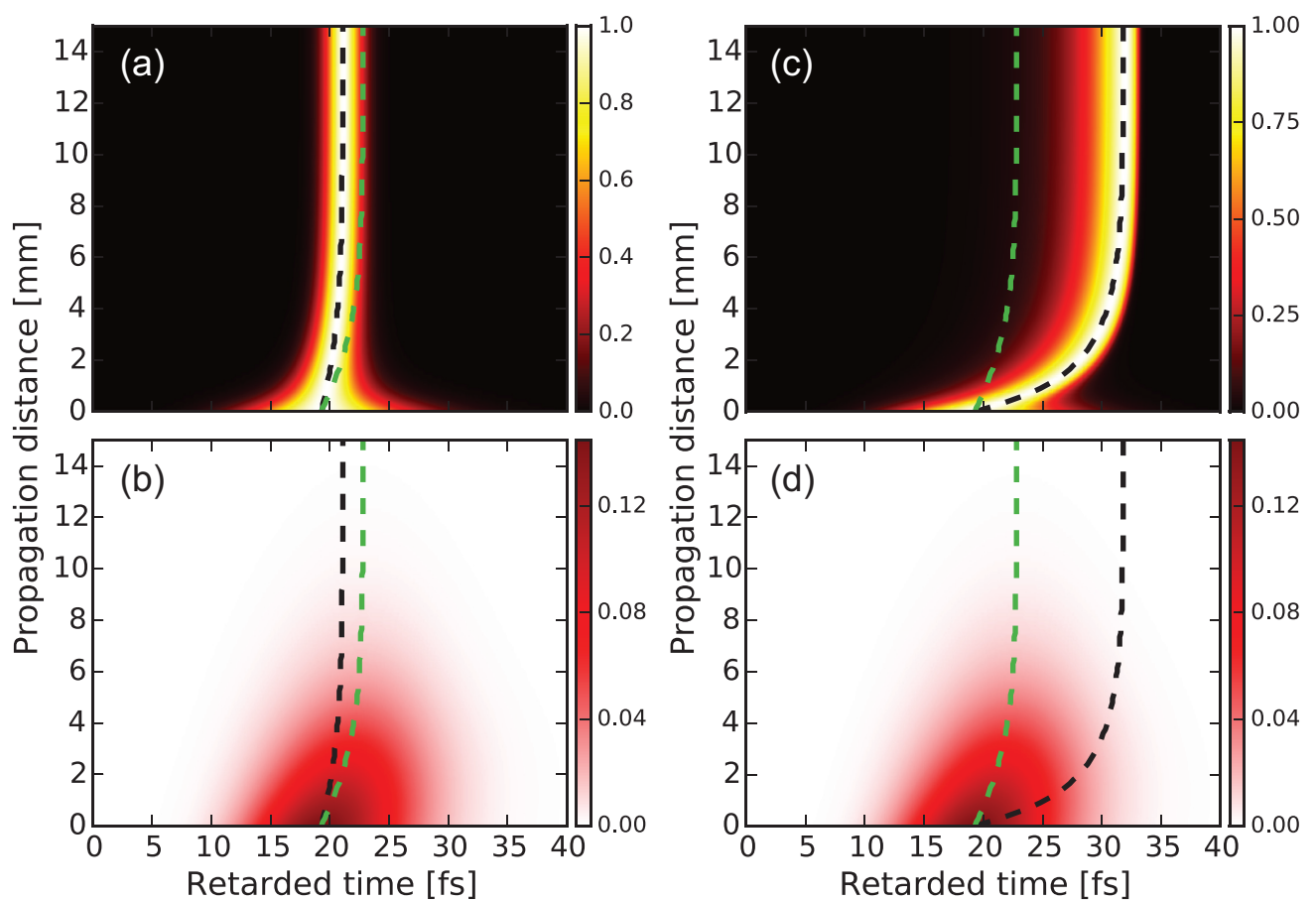

FIG. 8. (Color online) Comparison between (left) the rate equation approach and (right) the modified rate equations with the gain-dependent group velocity for a 10-fs pump pulse. (a) and (c) The normalized XRL intensity and (b) and (d) the population inversion as a function of retarded time and propagation distance. The black dashed line follows the maximum of the XRL intensity, while the green (gray) dashed line follows the maximum of the population inversion.
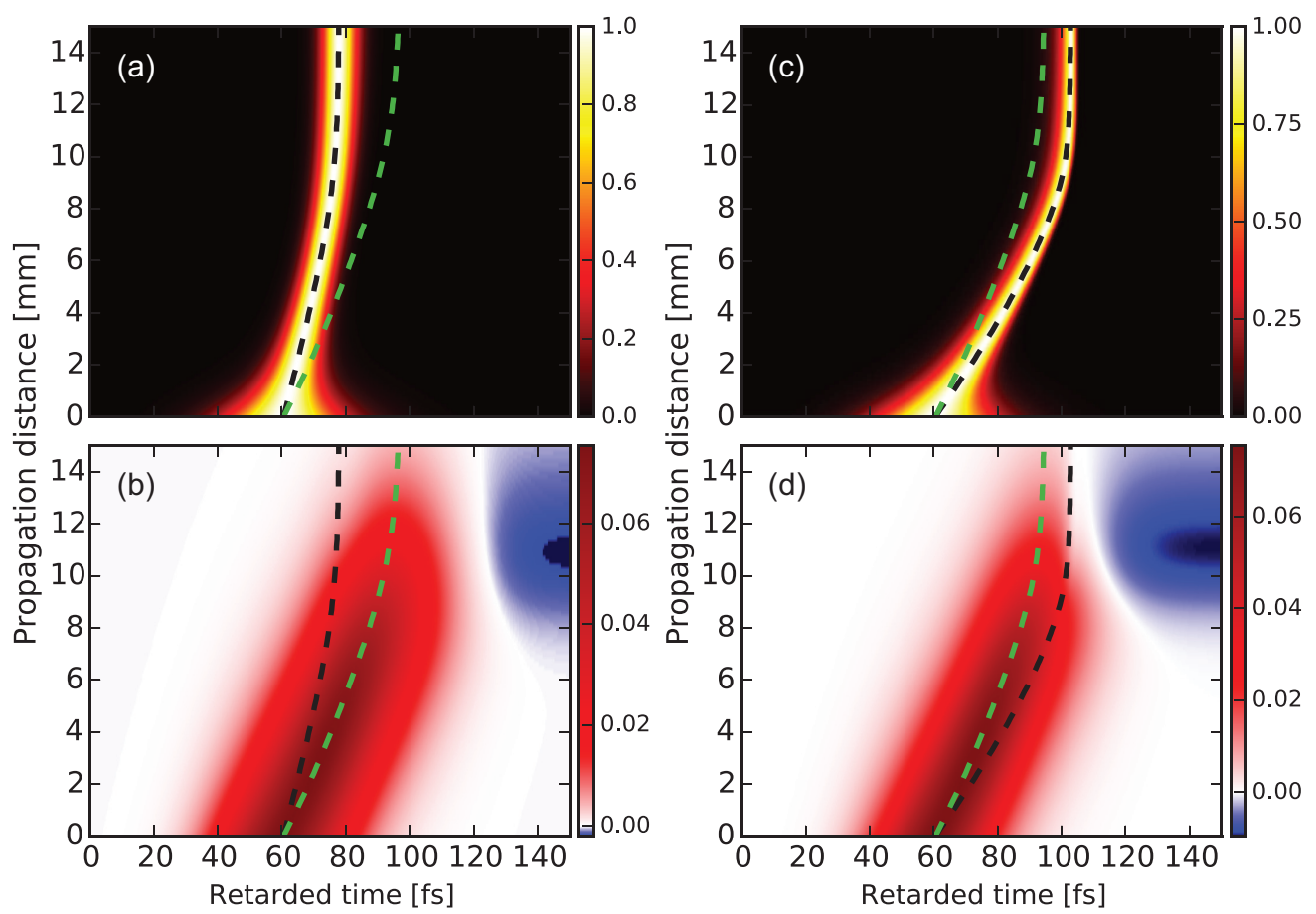

FIG. 9. (Color online) Comparison between (left) the rate equation approach and (right) the modified rate equations with the gain-dependent group velocity for a 40-fs pump pulse. (a) and (c) The normalized XRL intensity and (b) and (d) the population inversion as a function of retarded time and propagation distance. The black dashed line follows the maximum of the XRL intensity, while the green (gray) dashed line follows the maximum of the population inversion. 
the stimulated emission cross section. Treating the emitted radiation as intensity compared to the complex electric field in the Maxwell-Bloch approach therefore comes with a loss of spectral information.

We will show a comparison for two regimes: pump-pulse durations considerably longer than the Auger lifetime of the upper state and pulse durations on the order of the Auger lifetime. Figure 7 shows the gain curves for a pump pulse of 10 - and 40-fs pulse duration and compares rate equations to the Maxwell-Bloch approach. Figures 8(a) and 8(b) and 9(a) and 9(b) show the XRL intensity and the population inversion in the retarded frame as a function of propagation distance as determined by the rate-equation approach introduced in Ref. [15]. For a 10-fs Gaussian pulse the rate-equation model overestimates the number of XRL photons by an order of magnitude compared to the Maxwell-Bloch approach. The rate-equation model does not include the gain-dependent group velocity of the XRL. For a 10-fs pulse, the population inversion and the XRL intensity have an almost perfect temporal overlap (see Fig 8). This leads to an overestimation of the gain for the rate-equation approach.

For pulse durations much longer than the Auger decay the maximum of the population inversion propagates with a speed slower than the speed of light. This can be seen in Fig. 9(b) and is due to absorption of the leading edge of the XFEL pulse. Considerable slippage of the population inversion and the XRL pulse is observed. As a result the rate-equation approach underestimates the gain by 2 orders of magnitude for a $40-\mathrm{fs}$ pump pulse.

Group-velocity effects are not included in the rate-equation approach, and the amplification is directly modeled by the population inversion and not the macroscopic polarization. Depending on the pulse duration, this effect can either lead to an overestimation of the gain for short pump pulses or an underestimation of the gain for long pump pulses. A possible ad hoc improvement of the rate-equation model was suggested in Ref. [40] by explicitly introducing a gain-dependent group velocity for the emitted XRL radiation estimated by Eq. (5) of Ref. [35]:

$$
v_{g}=c[1+\operatorname{cg}(z, t) / \Gamma]^{-1},
$$

where $g(z, t)$ is the gain and $\Gamma$ is the upper-state lifetime. This equation for the group velocity was derived for a Doppler or homogeneously broadened medium. Comparing the group velocity obtained from our Maxwell-Bloch approach with that obtained from [35] shows only a qualitative agreement. Adding the gain-dependent group velocity term of Eq. (8) to the rate equation as done in [40] does not improve the gain estimation for the rate-equation approach. The gain-dependent group-velocity correction consistently overestimates the delay with propagation when it is compared to the Maxwell-Bloch approach, as shown in Figs. 8(c) and 8(d) and 9(c) and 9(d).

We hence conclude that the rate-equation approach gives only a rough estimate of the total output power of the transient photoionization-pumped x-ray laser. The ad hoc fix of adding a gain-dependent group velocity to the equation describing the evolution of the emitted XRL intensity does not improve the overall quality of the rate-equation approach. An inherent deficiency of the rate-equation approach that treats the amplification of the emitted time-dependent intensity profile and not the electric-field envelope is the lack of treating spectral changes that can occur during the propagation and amplification process. Since for transient $x$-ray laser amplifiers, in contrast to steady-state amplifiers, a time-dependent treatment of the emitted intensity is necessary, this approach does not allow any ad hoc corrections that could be implemented in a frequency-domain description. Correct modeling of the transient photoionization $\mathrm{X}$-ray laser therefore requires the solution of generalized Maxwell-Bloch equations.

\section{SELF-AMPLIFIED SPONTANEOUS-EMISSION PUMP PULSE}

Currently, most XFELs are based on self-amplified spontaneous emission (SASE) [41,42]. The pulses produced by the SASE process have a huge shot-to-shot fluctuation [43] of the pulse energy and spectrum and are characterized by low temporal coherence and a relatively large spectral bandwidth of around $1 \%$ in the soft $x$-ray regime $[43,44]$. The previous XRL experiments were performed in SASE operation, and we will hence characterize the XRL output for SASE pulses. In our theoretical approach, the SASE pulses were modeled as Gaussian noise [24,45], with a Gaussian power spectrum
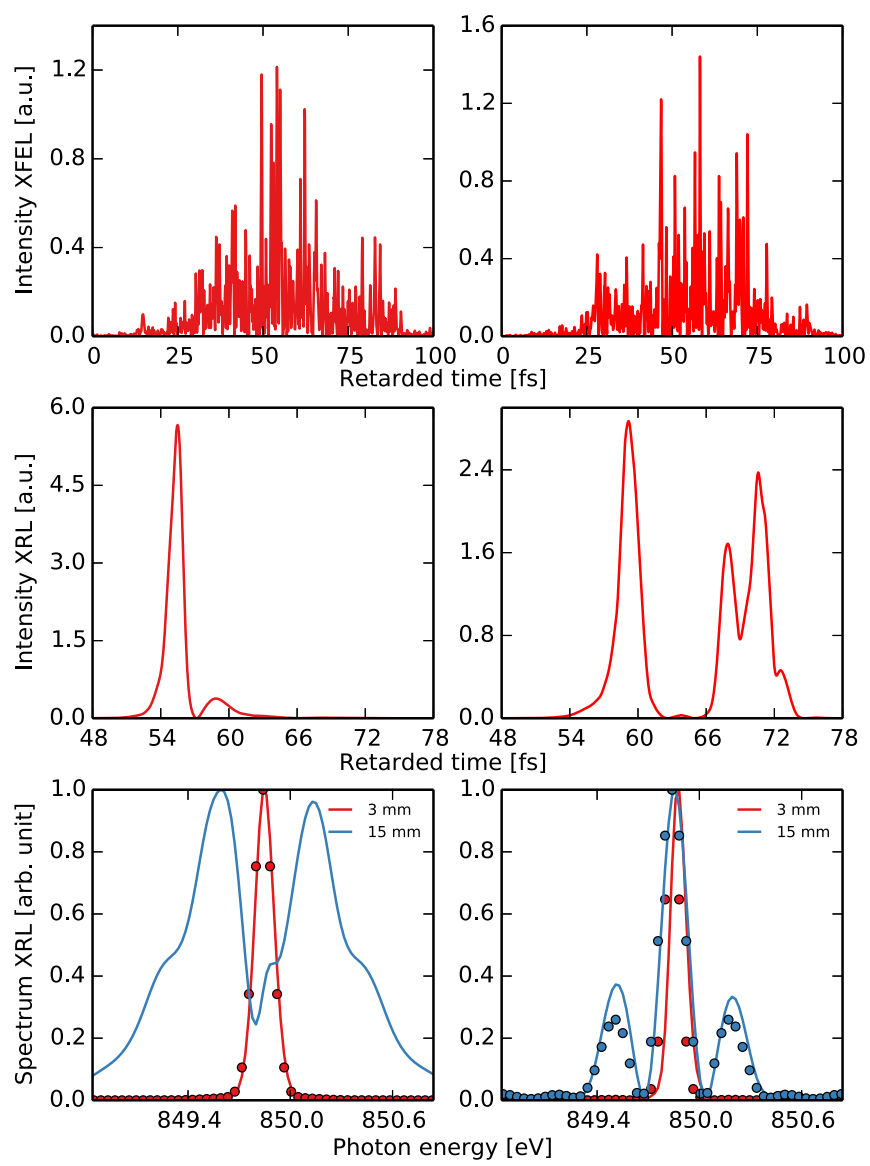

FIG. 10. (Color online) Comparison of two single SASE shots on the left and right. The top row shows the initial temporal profiles of the SASE shots. The middle row shows the final temporal profile of the XRL at the end of the medium. The bottom row shows the spectral intensity at two different points in the medium along with the test spectra (points). 

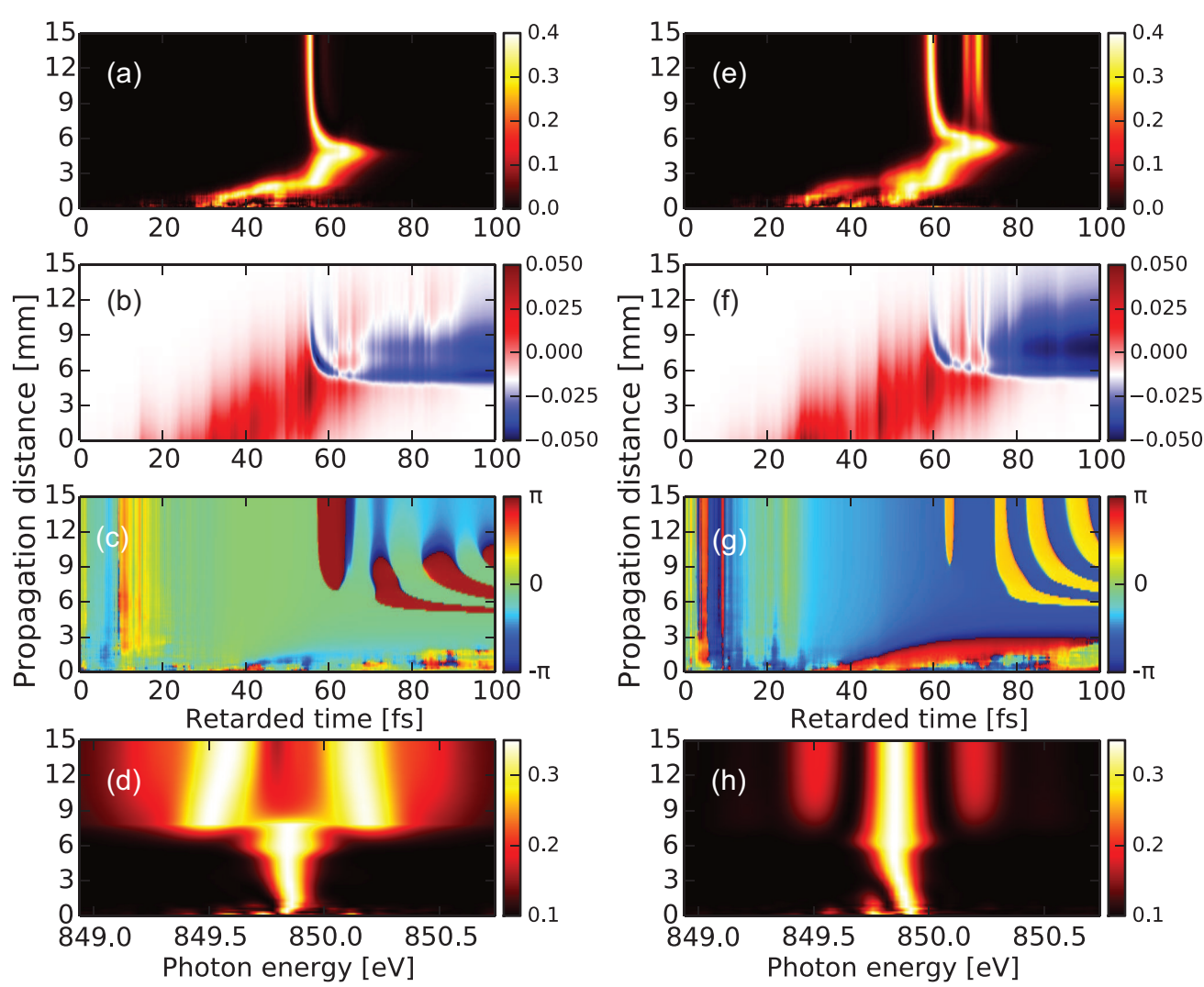

FIG. 11. (Color online) Evolution of (a) and (e) the normalized XRL intensity, (b) and (f) the population inversion $\rho_{1 s 1 s}-\rho_{2 p 2 p}$, (c) and (g) the phase of the XRL electric field, and (d) and (h) the XRL spectrum with propagation for the two different SASE shots in Fig. 10.

of $7 \mathrm{eV}$ (FWHM) width, corresponding to the experimental conditions of Ref. [2]. The temporal envelope was chosen to be Gaussian as well, with a pulse duration of $40 \mathrm{fs}$. According to the typical conditions of our experiment, we assume $2 \times 10^{12}$ photons per pulse, with a focus radius of $2 \mu \mathrm{m}$. We compare the results of two realizations of a SASE pulse. The two initial SASE pulses before they enter the gain medium are shown in the top row of Fig. 10. Although the pulses look similar and show no dominating features, the resulting XRL pulses show completely different characteristics. The resulting XRL intensity profile at the end of the gas medium is shown in the middle row of Fig. 10. The left XRL pulse shows a single main spike with high intensity, while the one on the right shows a multipeak structure with lower intensity. The bottom row of Fig. 10 depicts the corresponding spectra of the XRL pulses at two different points in the medium. To test if the XRL pulses are transform limited we compare the spectrum (Fourier transform of the complex field) with the Fourier transform of the absolute value of the field $I_{\text {test }}(\omega)=\int|\mathcal{E}|^{2} d t$ assuming a constant phase. In the middle of the exponential gain region the test spectra (dots) coincide with the actual spectra (solid lines), confirming that the XRL pulses are indeed transform limited, despite pumping with SASE pulses with low temporal coherence. At the end of the medium the XRL line is saturated, and Rabi broadening leads to side peaks in the spectrum. These are more pronounced on the left side since the single-spike pulse has a higher intensity. The left pulse is, strictly speaking, not transform limited at the end of the medium. The deviation, however, results from the coherent Rabi oscillations in the output pulse. These oscillations come with a phase change of $\pi$ between peaks [see Fig. 11(c)], which is not included in the test spectrum. Figures 11(a) and 11(e) show the temporal profile of the XRL as a function of propagation length for the two different SASE pulses. The plots show that both SASE shots initially produce XRLs with similar pulse shapes and spectra. But the pulses are strongly reshaped in the deep saturation region $[46,47]$, and due to their different peak intensities, the spectra and pulse shapes look very different. After saturation, the Rabi flopping (amplification of a $\pi$ pulse) leads to a ringing pattern and produces multiple temporal spikes in the time domain. This can be seen in Figs. 11(c) and 11(g), which show the phase of the XRL electric field. The different peaks are phase shifted by $\pi$. The left pulse with the strong intensity, however, loses the constant phase relation between the peaks with propagation. This reshaping of the phase with propagation makes the pulse strictly not transform limited.

The important conclusion here is that although the temporal profiles of the emitted XRL pulses vary from shot to shot, they are basically coherent (no random phase jumps), despite pumping with incoherent SASE pulses.

Since the output of the XRL varies from SASE pulse to SASE pulse, it is interesting to summarize some statistical properties of the ensemble of pulses generated by a SASE ensemble of pump pulses. Figure 12 shows that most of the generated $x$-ray pulses have a multipeak structure with around two to three temporal spikes. The temporal position of the highest-intensity peak also shows a very broad distribution with a width of $15 \mathrm{fs}$. 

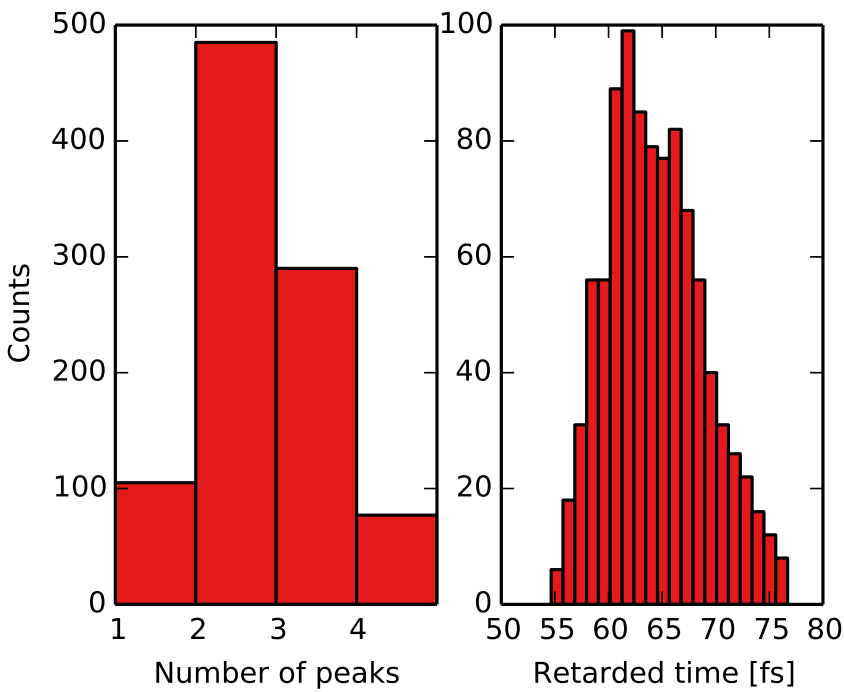

FIG. 12. (Color online) Distribution of the number of temporal peaks in the XRLs and time of the main peak for a SASE ensemble of 2000 shots. The incoming FEL pulse was centered at 65 fs.

To study the coherence of the generated $\mathrm{x}$-ray pulse we look at the first-order temporal correlation function, defined by

$$
g^{(1)}\left(t_{1}, t_{2}\right)=\frac{\left\langle E\left(t_{1}\right) E^{\star}\left(t_{2}\right)\right\rangle}{\sqrt{\left\langle\left|E\left(t_{1}\right)\right|^{2}\right\rangle\left\langle\left|E\left(t_{2}\right)\right|^{2}\right\rangle}},
$$

where the angle brackets $\langle\cdot\rangle$ denote the ensemble average. The correlation function is depicted in Fig. 13(a). The correlation function for a nonstationary pulse depends on $t_{1}$ and $t_{2}$ and not just the delay $\tau=t_{1}-t_{2}$. To study the coherence we look at cuts perpendicular to the main diagonal at the maximum intensity (blue line), which are a function of the delay $\tau$. There is no general definition for the coherence time for nonstationary distributions. In the following we will use the width of the first peak of $g^{(1)}(\tau)$ as a definition for the coherence time. At $3 \mathrm{~mm} g^{(1)}(\tau)$ is characterized by a single Gaussian function with a coherence time of $25 \mathrm{fs}$. The coherence time is strongly reduced at $6 \mathrm{~mm}$, but $g^{(1)}(\tau)$ shows two additional peaks. The additional maxima in the correlation function come from the multiple temporal peaks due to Rabi flopping and confirm that these peaks are actually
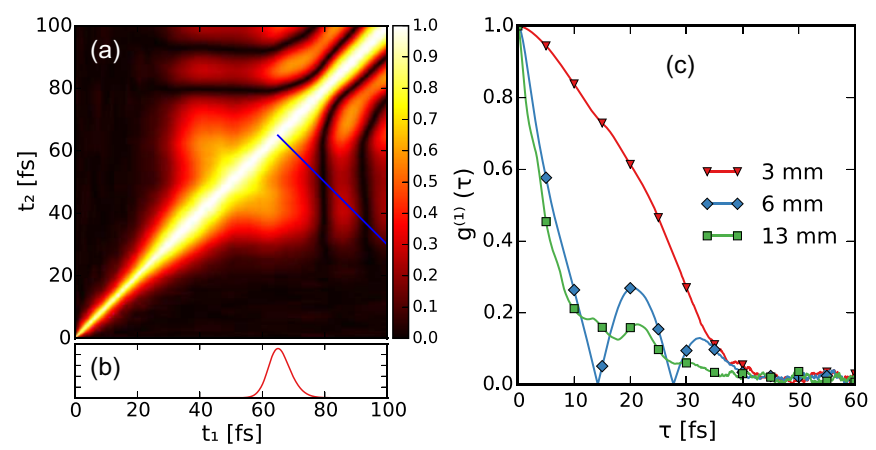

FIG. 13. (Color online) (a) The absolute value of the correlation function $\left|g^{(1)}\right|$ after $6 \mathrm{~mm}$ of propagation. (b) The average XRL intensity. (c) A cut through $g^{(1)}$ along the blue line in (a), $t_{1}-t_{2}$, for three different points along the medium.

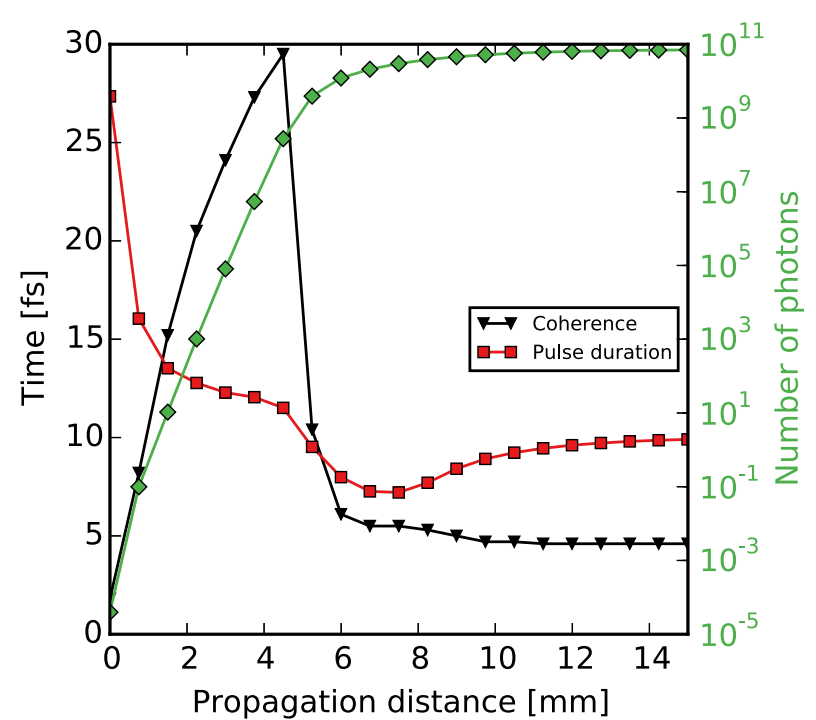

FIG. 14. (Color online) Evolution of the coherence time (black line with triangles), the averaged pulse duration (red line with squares) and number of photons in the XRL (green line with diamonds) with propagation distance for an ensemble of 2000 SASE pump pulses.

phase correlated. At $13 \mathrm{~mm}$ of propagation these peaks in $g^{(1)}$ disappear because the phase correlation between the intensity peaks in the individual pulses is lost by propagation in the saturation regime [see Fig. 11(c)].

The coherence time of $g^{(1)}(\tau)$ as a function of propagation distance along with the XRL photon number is depicted in Fig. 14. It shows an increases in the coherence until saturation sets in and then shows a quick drop in the temporal coherence. In the linear gain regime the coherence time exceeds the pulse duration, confirming the generation of fully coherent transform-limited $\mathrm{x}$-ray pulses.

\section{CONCLUSION}

In this paper we presented Maxwell-Bloch calculations for amplified $K \alpha$ florescence in neon based on two previous experiments $[1,2]$ and presented the evolution of the temporal and spectral intensity of the emitted radiation as a function of gain length. We considered the case of pumping with a coherent, transform-limited Gaussian pulse, which can be achieved by soft $x$-ray self-seeding schemes at XFEL facilities and the case of pumping by SASE pulses. The buildup of the gain is very similar in both cases. The amplification starts from spontaneous emission, and initially, the pulses are not transform limited. For the first gain lengths, gain narrowing is observed, along with a decrease of the XRL pulse duration, and a transform-limited pulse is created. This transform-limited pulse is further exponentially amplified in a soliton-like way, so that the spectral and temporal widths of the emitted pulse stay constant over a large gain length. At saturation the pulses get broadened by nonlinear effects, and Rabi flopping between the core-excited and the final state can be observed. An analysis of the temporal shape of the propagating pump pulse, the population inversion, and the emitted line emission as a function of propagation distance through the medium was presented. This analysis led to the discovery 
of a gain-guiding mechanism. A stabilization between the temporal overlap of the population inversion and the emitted $\mathrm{XRL}$ pulse was discovered that is due to an interplay of the gain-dependent group velocity of the XRL radiation and the temporal retardation of the population inversion caused by the pump-pulse absorption. A thorough comparison of the Maxwell-Bloch approach to the more common rateequation approach was presented. The gain estimates for both approaches varied by orders of magnitude depending on the pump-pulse duration. This underlines the necessity for using a Maxwell-Bloch approach rather than a rate-equation approach to model transient $x$-ray lasing schemes.

Our one-dimensional Maxwell-Bloch description shows excellent agreement with experiments [2] in terms of the total number of emitted x-ray photons and XFEL transmission. In order to predict spatial emission properties such as beam divergence, mode structure, and the transverse coherence a treatment of the transverse dimension is necessary. A complete theoretical characterization of the longitudinal coherence properties would also require treatment of the transverse direction, which was beyond the scope of this study.

\section{ACKNOWLEDGMENTS}

We would like to thank S.-K. Son for the calculation of the electronic dipole moments and V. Kimberg for stimulating discussions.
[1] N. Rohringer et al., Nature (London) 481, 488 (2012).

[2] C. Weninger, M. Purvis, D. Ryan, R. A. London, J. D. Bozek, C. Bostedt, A. Graf, G. Brown, J. J. Rocca, and N. Rohringer, Phys. Rev. Lett. 111, 233902 (2013).

[3] M. A. Duguay and P. M. Rentzepis, Appl. Phys. Lett. 10, 350 (1967).

[4] Y. Kato, Inst. Phys. Conf. Ser. 186, 3 (2005).

[5] J. J. Rocca, Rev. Sci. Instrum. 70, 3799 (1999).

[6] S. Suckewer and P. Jaegle, Laser Phys. Lett. 6, 411 (2009).

[7] S. Suckewer, C. H. Skinner, H. Milchberg, C. Keane, and D. Voorhees, Phys. Rev. Lett. 55, 1753 (1985).

[8] D. L. Matthews et al., Phys. Rev. Lett. 54, 110 (1985).

[9] T. S. Axelrod, Phys. Rev. A 13, 376 (1976).

[10] T. S. Axelrod, Phys. Rev. A 15, 1132 (1977).

[11] P. L. Hagelstein, Lawrence Livermore Lab., Report No. UCID17629 (1977).

[12] P. L. Hagelstein, Plasma Phys. 25, 1345 (1983).

[13] P. L. Hagelstein, Nineth International Conference on Atomic Physics, Seattle Washington (Lawrence Livermore National Laboratory, Livermore, CA, 1985), Report No. 92336.

[14] H. C. Kapteyn, Appl. Opt. 31, 4931 (1992).

[15] N. Rohringer and R. London, Phys. Rev. A 80, 013809 (2009).

[16] M. Ribière et al., Appl. Phys. B 101, 753 (2010).

[17] W. T. Silfvast, J. J. Macklin, and O. R. Wook II, Opt. Lett. 8, 551 (1983).

[18] H. C. Kapteyn, R. W. Lee, and R. W. Falcone, Phys. Rev. Lett. 57, 2939 (1986).

[19] V. Ayvazyan et al., Eur. Phys. J. D 37, 297 (2006).

[20] P. Emma et al., Nat. Photon. 4, 641 (2010).

[21] T. Ishikawa et al., Nat. Photon. 6, 540 (2012).

[22] E. Allaria et al., Nat. Photon. 6, 699 (2012).

[23] W. Ackermann et al., Nat. Photon. 1, 336 (2007).

[24] C. Weninger and N. Rohringer, Phys. Rev. A 88, 053421 (2013).
[25] F. A. Hopf and P. Meystre, Phys. Rev. A 12, 2534 (1975).

[26] F. Hopf, P. Meystre, and D. W. McLaughlin, Phys. Rev. A 13, 777 (1976).

[27] O. Larroche, D. Ros, A. Klisnick, A. Sureau, C. Möller, and H. Guennou, Phys. Rev. A 62, 043815 (2000).

[28] C. M. Kim, K. A. Janulewicz, and J. Lee, Phys. Rev. A 84, 013834 (2011).

[29] B. Fidel, E. Heyman, R. Kastner, and R. Ziolkowski, J. Comput. Phys. 138, 480 (1997).

[30] N. Rohringer and R. Santra, Phys. Rev. A 86, 043434 (2012).

[31] B. Bidégaray, A. Bourgeade, and D. Reignier, J. Comput. Phys. 170, 603 (2001).

[32] J. Amann et al., Nat. Photon. 6, 693 (2012).

[33] L. W. Casperson, J. Appl. Phys. 48, 256 (1977).

[34] G. J. Pert, J. Opt. Soc. Am. B 11, 1425 (1994).

[35] L. Casperson and A. Yariv, Phys. Rev. Lett. 26, 293 (1971).

[36] R. Tommasini and E. Fill, Phys. Rev. A 62, 034701 (2000).

[37] F. Strati and G. J. Tallents, Phys. Rev. A 64, 013807 (2001).

[38] F. A. Hopf, P. Meystre, M. O. Scully, and J. F. Seely, Phys. Rev. Lett. 35, 511 (1975).

[39] F. A. Hopf, Phys. Rev. A 30, 3336 (1984).

[40] Q. Miao, J.-C. Liu, H. Ågren, J.-E. Rubensson, and F. Gel'mukhanov, Phys. Rev. Lett. 109, 233905 (2012).

[41] R. Bonifacio, C. Pellegrini, and L. Narducci, Opt. Commun. 50, 373 (1984).

[42] E. Saldin, E. Schneidmiller, and M. Yurkov, Opt. Commun. 148, 383 (1998).

[43] A. M. Kondratenko and E. L. Saldin, Part. Accel. 10, 207 (1980).

[44] S. Krinsky and R. L. Gluckstern, Phys. Rev. Spec. Top. Accel. Beams 6, 050701 (2003).

[45] G. Vannucci and M. C. Teich, Appl. Opt. 19, 548 (1980).

[46] G. L. Lamb, Rev. Mod. Phys. 43, 99 (1971).

[47] J.-Z. Zhang and I. Galbraith, J. Appl. Phys. 96, 922 (2004). 